\title{
A Novel Homozygous p.L539F Mutation Identified in PINK1 Gene in a Moroccan Patient with Parkinsonism
}

\author{
Rafiqua Ben El Haj, ${ }^{1}$ Wafaa Regragui, ${ }^{1,2}$ Rachid Tazi-Ahnini, ${ }^{3}$ Asmae Skalli, ${ }^{1}$ \\ Naima Bouslam, ${ }^{2}$ Ali Benomar, ${ }^{1,2}$ Mohamed Yahyaoui, ${ }^{1,2}$ and Ahmed Bouhouche ${ }^{1,2}$ \\ ${ }^{1}$ Research Team on Neurodegenerative Diseases, Medical School and Pharmacy, Mohammed V University, 10100 Rabat, Morocco \\ ${ }^{2}$ Department of Neurology and Neurogenetics, Specialties Hospital, CHU Ibn Sina, 10100 Rabat, Morocco \\ ${ }^{3}$ Laboratory of Biotechnology, Medical School and Pharmacy, Mohammed V University, 10100 Rabat, Morocco
}

Correspondence should be addressed to Ahmed Bouhouche; a.bouhouche@um5s.net.ma

Received 2 March 2016; Revised 4 May 2016; Accepted 16 May 2016

Academic Editor: Enza M. Valente

Copyright (C) 2016 Rafiqua Ben El Haj et al. This is an open access article distributed under the Creative Commons Attribution License, which permits unrestricted use, distribution, and reproduction in any medium, provided the original work is properly cited.

\begin{abstract}
Parkinson's disease (PD) is the second most common neurodegenerative disorder after Alzheimer's disease. Ten of fifteen causative genes linked to familial forms of PD have been reported to cause autosomal recessive forms. Among them, mutations in the PTEN-induced kinase 1 (PINK1) gene were shown to be responsible for a phenotype characterized by early onset, good response to levodopa, and a benign course. Using chromosomal microarray analysis and Sanger sequencing, we identified a homozygous G/C substitution in a 58-year-old Moroccan man diagnosed with recessive inherited Parkinson's disease. This G-to-C transition occurred at position 1617 leading to an amino acid change L/F at position 539 located in highly conserved motif in the $\mathrm{C}$ terminal sequence of PINK1. Interestingly, the c.1617G >C substitution is absent in 192 ethnically matched control chromosomes. Our findings have shown that the p.L539F is a novel mutation located in the $\mathrm{C}$ terminal sequence of the PINK1 protein that could be pathogenic and responsible for a clinical phenotype resembling idiopathic Parkinson's disease with rapid progression and early cognitive impairment.
\end{abstract}

\section{Introduction}

Parkinson's disease (PD) is a complex, chronic, and progressive neurodegenerative disease characterized by the classic triad that includes rest tremor, rigidity, and bradykinesia as motor symptoms [1]. The PD prevalence is age-related; it is about $1-2 \%$ concerning elderly people over 65 years and can reach $4-5 \%$ for subjects over 85 years of age [2]. During the course of disease evolution, secondary symptoms can occur such as motor and nonmotor symptoms leading to a real disability [3]. This symptom array results from a significant dopaminergic neurodegeneration in certain brain areas, especially in the substantia nigra pars compacta [4]. The first PD clinical manifestations occur only when the dopaminergic neurons loss reaches at least 50\% [5].

The etiology of PD is likely to be multifactorial involving genetic and environmental factors as well as brain aging process. Nevertheless, several variants in PARK loci have been associated with rare Mendelian forms of PD. Among them, there are at least 10 different genes associated with autosomal recessive forms: PRKN (PARK2), PINK1 (PARK6), DJ1 (PARK7), ATP13A2 (PARK9), PLA2G6 (PARK14), FBXO7 (PARK15), DNAJC6 (PARK19), and SYNJ1 (PARK20) [6] and recently $P O D L X$ and $V P S 13 C$ genes $[7,8]$. Mutations in the $P R K N$ gene, including point mutations and exon rearrangements, are the most common and account for up to 50\% and $15 \%$ of familial autosomal recessive and sporadic early onset $\mathrm{PD}$, respectively. $P R K N$ phenotype is indistinguishable from idiopathic PD with good response to levodopa and a benign course [9]. PINK1 gene mutations are the second most frequent in familial early onset $\mathrm{PD}$, compatible with recessive inheritance, and explain 1-9\% of cases depending on ethnic origin. The clinical phenotype is characterized by early onset, slow progression, and good levodopa response [10].

Approximately 50 mutations in PINK1 gene have been considered to be pathogenic in both sporadic and familial PD cases; exon 7 comprises the largest number of mutations found, with Q456X mutation as the most frequent one 
[11]. Most of these mutations induce a loss of function of the protein, thereby preventing the parkin recruitment and phosphorylation [12]. It is now clear that the combination of the activities of both proteins PINK1 and PRKN is essential for the mitochondrial function regulation and thus for cell survival, by conferring cells neuroprotective properties suggesting critical role of both proteins in the pathogenesis of PD [13].

In the present study, we report a novel homozygous missense mutation in exon 8 of PINK1 gene in a Moroccan PD patient with a rapid progression and a mild cognitive impairment.

\section{Patient and Methods}

2.1. Patient and Controls. The proband, the eldest in a family of five sibs, is a 58-year-old Moroccan man from a consanguineous marriage, referred to the Movement Disorder Unit of the Department of Neurology (Hôpital des Spécialités, Rabat, Morocco) in 2010. Ninety-six control individuals were recruited at the Blood Transfusion Center (Rabat, Morocco). Genomic DNA was extracted from peripheral blood leukocytes using Isolate II Genomic DNA Kit from Bioline. The study was approved by the local ethics committee and an informed written consent was obtained from all participants.

2.2. Chromosomal Microarray Analysis. The patient DNA sample was subjected to high resolution CMA using an Affymetrix platform and CytoScan HD microarrays according to the manufacturer's protocol. Briefly, $250 \mathrm{ng}$ of DNA samples was digested with Nsp1, amplified with TITANIUM Taq DNA polymerase (Clontech, Mountain View, CA), fragmented with Affymetrix fragmentation reagent, and labeled with biotin end-labeled nucleotides. The DNA was hybridized to the microarray for 16 hours, washed and stained on GeneChip Fluidics Station 450, and scanned on the GeneChip Scanner 3000 7G (Affymetrix). Data analysis was performed using Chromosome Analysis Suite software version 1.2.2 (Affymetrix). These chips include 750,000 SNP and 2.6 million $\mathrm{CNV}$ markers that enable high resolution (25-50 kb resolution) detection of CNV, regions of homozygosity $(\mathrm{ROH})$, uniparental disomy, and low-level mosaicism.

2.3. Candidate Gene Sequencing. Since the LRRK2 p.G2019S mutation is very common among PD patients from North Africa and consanguineous patients homozygous for the mutation have been reported [14], exon 41 of $L R R K 2$ gene was first sequenced in our patient. All the coding exons and intron-exon boundaries of PINK1 gene were then PCR amplified and the PCR products were sequenced using Big Dye Terminator Cycle Ready Reaction 3.1 Kits and an ABI 3130xl automated sequencer for the patient and the 96 controls. The collected sequence chromatograms were analyzed using SeqScape2.1 software (Applied Biosystems, Foster City, CA).

2.4. Bioinformatics Analysis. To predict the pathogenesis of PINK1 c.1617G>C mutation, the wild and mutant PINK1 cDNA sequences were both analyzed using MutationTaster
(I)

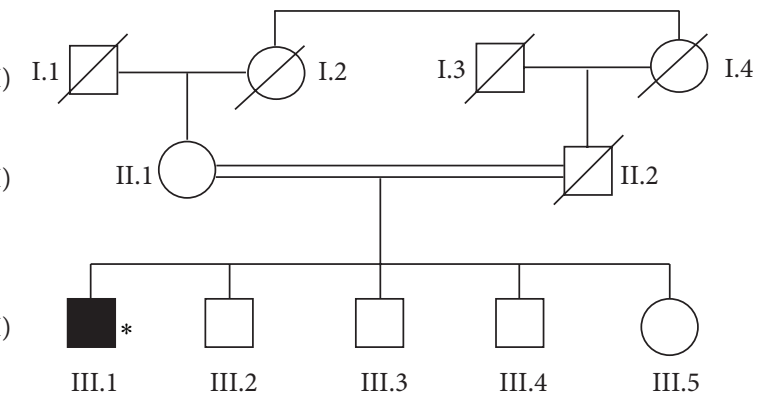

FIgure 1: Pedigree of family RBT-BOU-PAR. DNA sample was available from patient with asterisk.

software (http://www.mutationtaster.org/). This program calculates probabilities for the alteration to be either a disease mutation or a harmless polymorphism. Similarly, PINK1 amino acid sequences containing $\mathrm{L}$ or $\mathrm{F}$ at position 539 were analyzed using PolyPhen-2 (http://genetics.bwh.harvard.edu/ pph2/) and SIFT software (http://sift.jcvi.org/). Based on straightforward physical and comparative considerations, PolyPhen-2 predicts the possible impact of an amino acid substitution on the structure and function of human protein while SIFT is able to predict whether an amino acid substitution affects protein function.

\section{Results}

3.1. Clinical Report. Patient III.1, a 58-year-old man of Moroccan origin, is the eldest in a family of five from a consanguineous marriage of the first degree and without family history of Parkinsonism (Figure 1). His disease began at the age of 54 as a right akinetic-rigid syndrome with slight tremor in the hand. He also complained about neck pain and sleep disturbance (rapid eye movement behavior disorder). He was treated with the dopamine agonist piribedil at $150 \mathrm{mg}$ daily, with good evolution. Levodopa and amantadine were introduced few months later. Two years after disease onset, he complained about morning akinesia and wearing off that resolved with increasing doses of dopaminergic therapy up to $1000 \mathrm{mg}$ of levodopa equivalent dose and $200 \mathrm{mg}$ of amantadine per day. At the age of 58, he had an aggravation of motor fluctuations (delay on and 1 hour of efficacy) and the occurrence of dyskinesia without axial symptoms. The patient was evaluated for deep brain stimulation. MRI and psychiatric evaluation were normal and DOPA test was positive but neuropsychological evaluation revealed a Mattis Dementia Rating Scales score of 128/144. Therefore, a retest was scheduled for 6 months later and therapy adjustment was proposed.

3.2. High Resolution CMA. Analysis of allele difference plot of the entire genome given by high resolution CMA showed a normal karyotype with no pathogenic CNV but revealed the presence of many $\mathrm{ROH}$ through the 23 autosomes. At the 10 known loci for autosomal recessive $\mathrm{PD}$, only one $\mathrm{ROH}$ of 11.68 Mb was identified at chromosome $1 \mathrm{p}$ from bands $1 \mathrm{p} 36.13$ to p35.3 (Figure 2(a)) containing 244 genes including PINK1 gene. 


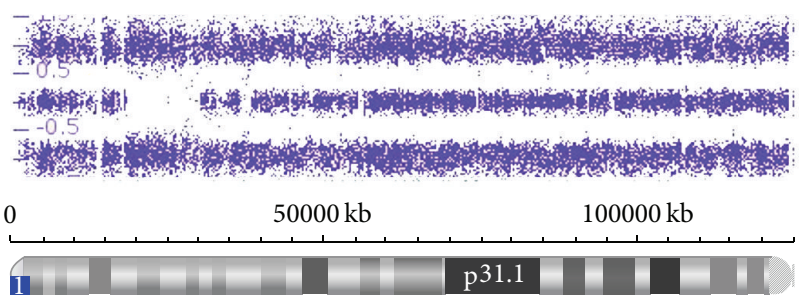

$\overline{1.68 \mathrm{M} b}$

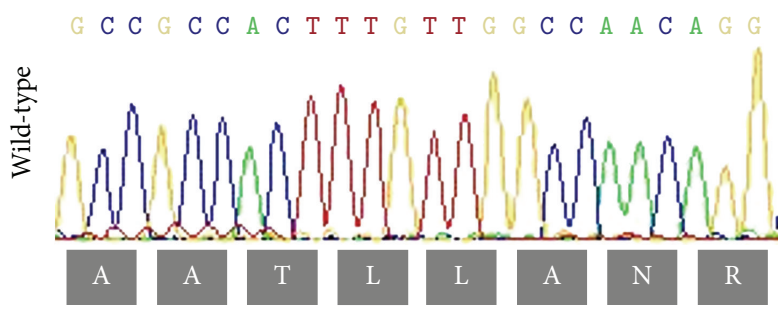

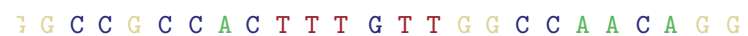

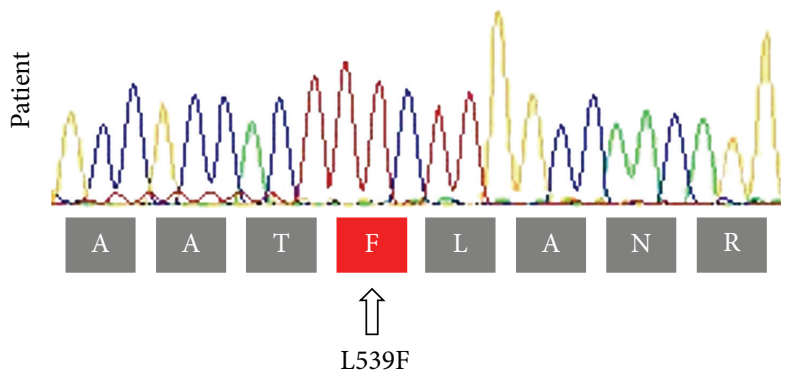

(b)

(a)

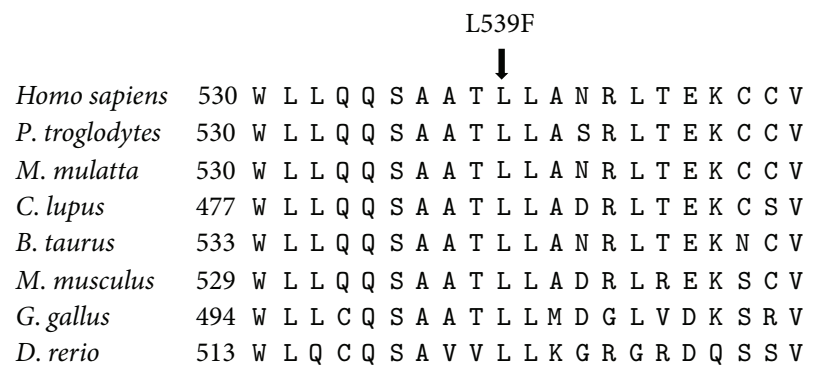

(c)

FIgure 2: Chromosomal microarray analysis, Sanger sequencing, and sequence alignment for PINK1 gene. (a) Allele difference plot of patient III.1 showing a ROH of $11.68 \mathrm{Mb}$ at PINK1 gene locus. (b) Sanger sequencing of PINK1 exon 8 showing the p.L539F mutation at homozygous state and the wild type sequence in a normal individual. (c) Multiple sequences alignment for human PINK1 gene. Conservation of amino acid $\mathrm{L}$ at position 539 among various species is indicated by arrow.

3.3. Candidate Gene Sequencing. Sequencing analysis of exon 41 of LRRK2 gene did not find the p.G2019S mutation, whereas sequencing of the PINK1 coding exons and intronexon boundaries revealed a novel homozygous G-to-C transition (Figure 2(b)) at nucleotide 1617 in PINK1 exon 8 (c.1617G $>$ C) resulting in a missense mutation of leucine to phenylalanine at amino acid residue 539 (p.L539F). DNA samples of the other family members were unfortunately not available for segregation analysis. However, the p.L539F mutation was absent from 192 ethnically matched control chromosomes. The substituted amino acid, located in the $\mathrm{C}$ terminal sequence region outside of the functional domains of the protein, is highly conserved among diverse group of species which indicates the importance of this amino acid. In addition, this mutation is located in a highly conserved amino acid motif from W530 to A541 (Figure 2(c)).

3.4. Bioinformatics Analysis. MutationTaster software analysis revealed that PINK1 c.1617G>C mutation could be a disease causing mutation with a score of 0.977. Analysis with PolyPhen-2 software of p.L539F mutation supports the probably damaging role of this amino acid change with a high score of 0.999 (sensitivity: 0.14; specificity: 0.99). Also, SIFT program gave a probability of 0.02 which predicted a deleterious effect.

\section{Discussion}

In our Moroccan consanguineous $\mathrm{PD}$ patient, the whole genome analysis by CMA did not reveal any pathogenic $\mathrm{CNV}$, including exon rearrangements $P R K N$ gene, the most common among autosomal recessive forms of PD [9]. However, sequencing of the only $\mathrm{ROH}$-based candidate gene showed a novel homozygous mutation p.L539F in exon 8 of PINK1 gene. Several evidences were in favor of the pathogenic effect of this novel p.L539F mutation: (1) the patient DNA shows $\mathrm{ROH}$ at only PINK1 locus among the 10 known recessive PD loci; (2) it is absent in 192 ethnically matched chromosomes; (3) it is not reported in publicly available variation databases accessed in February 2016 including dbSNP, ensembl, 1000 Genomes Project, Exome Aggregation Consortium (ExAC), and Exome Sequencing Project (ESP) databases; (4) the mutation affects a highly conserved amino acid motif; and (5) this mutation was predicted to be probably damaging 
by PolyPhen-2, damaging by SIFT, and disease causing by MutationTaster software. Previously, we have excluded the G2019S mutation of the LRRK2 gene, the most common PD mutation in the Maghreb [14]. Unfortunately, the GBA gene, a worldwide common risk factor for PD particularly in the Ashkenazi Jewish population [15], was not tested in our patient.

PINK1 is a protein of 581 amino acids, with an $\mathrm{N}$ terminal mitochondrial targeting sequence, a putative transmembrane domain, a serine/threonine kinase domain, and a $\mathrm{C}$ terminal noncatalytic region. The role of the $\mathrm{C}$ terminal of the protein is not well known, but it has been postulated that this protein tail could regulate PINK1 kinase activity [16-18] and could play a role in PINK1 targeting and submitochondrial localization [19]. Several PINK1 mutations have been reported and most of them are located in the protein kinase domain [20]. They are responsible for a clinical phenotype characterized by early onset, generally in the third-fourth decade (range 18-52 years), good response to levodopa, and slow progression but rapid onset of motor fluctuation and dyskinesia. The first symptom is most often tremor or foot dystonia, and additional signs such as sleep disturbance and psychiatric and cognitive impairments could appear during disease evolution [21-23]. In the C terminal tail of PINK1 gene, only three mutations at homozygous state were reported to be pathogenic. The p.519fsX522 mutation was reported in a patient with an age of onset of 40 years and no particular characteristics in addition to the classical clinical triad of PD [22]. Another frameshift truncating mutation p.D525fsX562 has been found in two patients resembling closely parkinrelated disease, with early onset (28 years), good levodopa response, and slow progression [24, 25]. Finally, a small insertion p.534_535insQ, located in the highly conserved amino acid motif (from W530 to A541) 4 nucleotides before our novel mutation, is responsible for early onset PD (32 years) with foot dystonia at onset and a good response to levodopa and without cognitive and psychiatric symptoms after a disease duration of 25 years [26].

The clinical presentation in our patient with this novel homozygous p.L539F mutation was typical with the classical clinical triad of Parkinson's disease but was slightly different from that due to the already reported PINK1 mutations. Indeed, the disease started relatively later at age 54, the cognitive impairment was early, and the disease progression was rapid since stimulation was proposed for the patient after only 4 years of disease duration.

\section{Conclusion}

We report in this study a novel homozygous missense mutation p.L539F in PINK1 gene that could be pathogenic in a Moroccan consanguineous patient and extended the phenotypic spectrum of PINK1-associated Parkinsonism. Further functional studies should be considered to confirm the pathogenicity of this novel mutation.

\section{Competing Interests}

The authors of this paper declare that they have no competing interests.

\section{Acknowledgments}

The authors are grateful to the patient for participation in this study. This work was supported by the "Centre National de Recherche Scientifique et Technique" (CNRST), "Ministère de l'Enseignement Supérieur, de la Recherche Scientifique et de la Formation des Cadres" (MESRSFC), and Mohammed V University in Rabat (UM5R), Morocco.

\section{References}

[1] R. Xia and Z.-H. Mao, "Progression of motor symptoms in Parkinson's disease," Neuroscience Bulletin, vol. 28, no. 1, pp. 3948, 2012.

[2] G. Alves, E. B. Forsaa, K. F. Pedersen, M. Dreetz Gjerstad, and J. P. Larsen, "Epidemiology of Parkinson's disease," Journal of Neurology, vol. 255, supplement 5, pp. 18-32, 2008.

[3] J. M. Shulman, P. L. De Jager, and M. B. Feany, "Parkinson's disease: genetics and pathogenesis," Annual Review of Pathology: Mechanisms of Disease, vol. 6, pp. 193-222, 2011.

[4] M. C. J. Dekker, V. Bonifati, and C. M. van Duijn, "Parkinson's disease: piecing together a genetic jigsaw," Brain, vol. 126, no. 8, pp. 1722-1733, 2003.

[5] K. Wirdefeldt, H.-O. Adami, P. Cole, D. Trichopoulos, and J. Mandel, "Epidemiology and etiology of Parkinson's disease: a review of the evidence," European Journal of Epidemiology, vol. 26, supplement 1, pp. S1-S58, 2011.

[6] M. K. Lin and M. J. Farrer, "Genetics and genomics of Parkinson's disease," Genome Medicine, vol. 6, no. 6, article 48, 2014.

[7] S. Sudhaman, K. Prasad, M. Behari, U. B. Muthane, R. C. Juyal, and B. K. Thelma, "Discovery of a frameshift mutation in podocalyxin-like (PODXL) gene, coding for a neural adhesion molecule, as causal for autosomal-recessive juvenile Parkinsonism," Journal of Medical Genetics, 2016.

[8] S. Lesage, V. Drouet, E. Majounie et al., "Loss of VPS13C function in autosomal-recessive parkinsonism causes mitochondrial dysfunction and increases pink1/parkin-dependent mitophagy," The American Journal of Human Genetics, vol. 98, no. 3, pp. 500513,2016

[9] V. Bonifati, "Genetics of Parkinson's disease-state of the art, 2013," Parkinsonism \& Related Disorders, vol. 20, supplement 1, pp. S23-S28, 2014.

[10] C. Klein and A. Westenberger, "Genetics of Parkinson's disease," Cold Spring Harbor Perspectives in Medicine, vol. 2, no. 1, article a008888, 2012.

[11] J. Siuda, B. Jasinska-Myga, M. Boczarska-Jedynak et al., "Earlyonset Parkinson's disease due to PINK1 p.Q456X mutationclinical and functional study," Parkinsonism and Related Disorders, vol. 20, no. 11, pp. 1274-1278, 2014.

[12] N. Exner, A. K. Lutz, C. Haass, and K. F. Winklhofer, "Mitochondrial dysfunction in Parkinson's disease: molecular mechanisms and pathophysiological consequences," The EMBO Journal, vol. 31, no. 14, pp. 3038-3062, 2012.

[13] S. Matsuda, Y. Kitagishi, and M. Kobayashi, "Function and characteristics of PINK1 in mitochondria," Oxidative Medicine and Cellular Longevity, vol. 2013, Article ID 601587, 6 pages, 2013.

[14] P. Gómez-Garre, F. Carrillo, and P. Mir, "Prevalence and clinical features of LRRK2 mutations in patients with Parkinson's Disease," European Neurological Journal, vol. 2, no. 2, pp. 1-7, 2010. 
[15] E. Sidransky, M. A. Nalls, J. O. Aasly et al., "Multicenter analysis of glucocerebrosidase mutations in Parkinson's disease," The New England Journal of Medicine, vol. 361, no. 17, pp. 1651-1661, 2009.

[16] C. H. Sim, D. S. S. Lio, S. S. Mok et al., "C-terminal truncation and Parkinson's disease-associated mutations down-regulate the protein serine/threonine kinase activity of PTEN-induced kinase-1," Human Molecular Genetics, vol. 15, no. 21, pp. 32513262, 2006.

[17] R. D. Mills, C. H. Sim, S. S. Mok, T. D. Mulhern, J. G. Culvenor, and H.-C. Cheng, "Biochemical aspects of the neuroprotective mechanism of PTEN-induced kinase-1 (PINK1)," Journal of Neurochemistry, vol. 105, no. 1, pp. 18-33, 2008.

[18] H. I. Woodroof, J. H. Pogson, M. Begley et al., "Discovery of catalytically active orthologues of the Parkinson's disease kinase PINK1: analysis of substrate specificity and impact of mutations," Open Biology, vol. 1, no. 3, Article ID 110012, 2011.

[19] D. Becker, J. Richter, M. A. Tocilescu, S. Przedborski, and W. Voos, "Pinkl kinase and its membrane potential $(\Delta \psi)$ dependent cleavage product both localize to outer mitochondrial membrane by unique targeting mode," The Journal of Biological Chemistry, vol. 287, no. 27, pp. 22969-22987, 2012.

[20] S. Kawajiri, S. Saiki, S. Sato, and N. Hattori, "Genetic mutations and functions of PINK1," Trends in Pharmacological Sciences, vol. 32, no. 10, pp. 573-580, 2011.

[21] S. A. Schneider and C. Klein, "PINK1 type of young-onset Parkinson disease,", in GeneReviews ${ }^{\circledR}, 2010$.

[22] P. Ibáñez, S. Lesage, E. Lohmann et al., "Mutational analysis of the PINK1 gene in early-onset parkinsonism in Europe and North Africa," Brain, vol. 129, no. 3, pp. 686-694, 2006.

[23] L. Ricciardi, S. Petrucci, A. Guidubaldi et al., "Phenotypic variability of PINK1 expression: 12 Years' clinical follow-up of two Italian families," Movement Disorders, vol. 29, no. 12, pp. 1561-1566, 2014.

[24] C. F. Rohé, P. Montagna, G. Breedveld, P. Cortelli, B. A. Oostra, and V. Bonifati, "Homozygous PINK1 C-terminus mutation causing early-onset parkinsonism," Annals of Neurology, vol. 56, no. 3, pp. 427-431, 2004.

[25] V. Bonifati, C. F. Rohé, G. J. Breedveld et al., "Early-onset parkinsonism associated with PINK1 mutations: frequency, genotypes, and phenotypes," Neurology, vol. 65, no. 1, pp. 87-95, 2005.

[26] C. Klein, A. Djarmati, K. Hedrich et al., "PINK1, Parkin, and DJ1 mutations in Italian patients with early-onset parkinsonism," European Journal of Human Genetics, vol. 13, no. 9, pp. 10861093, 2005. 

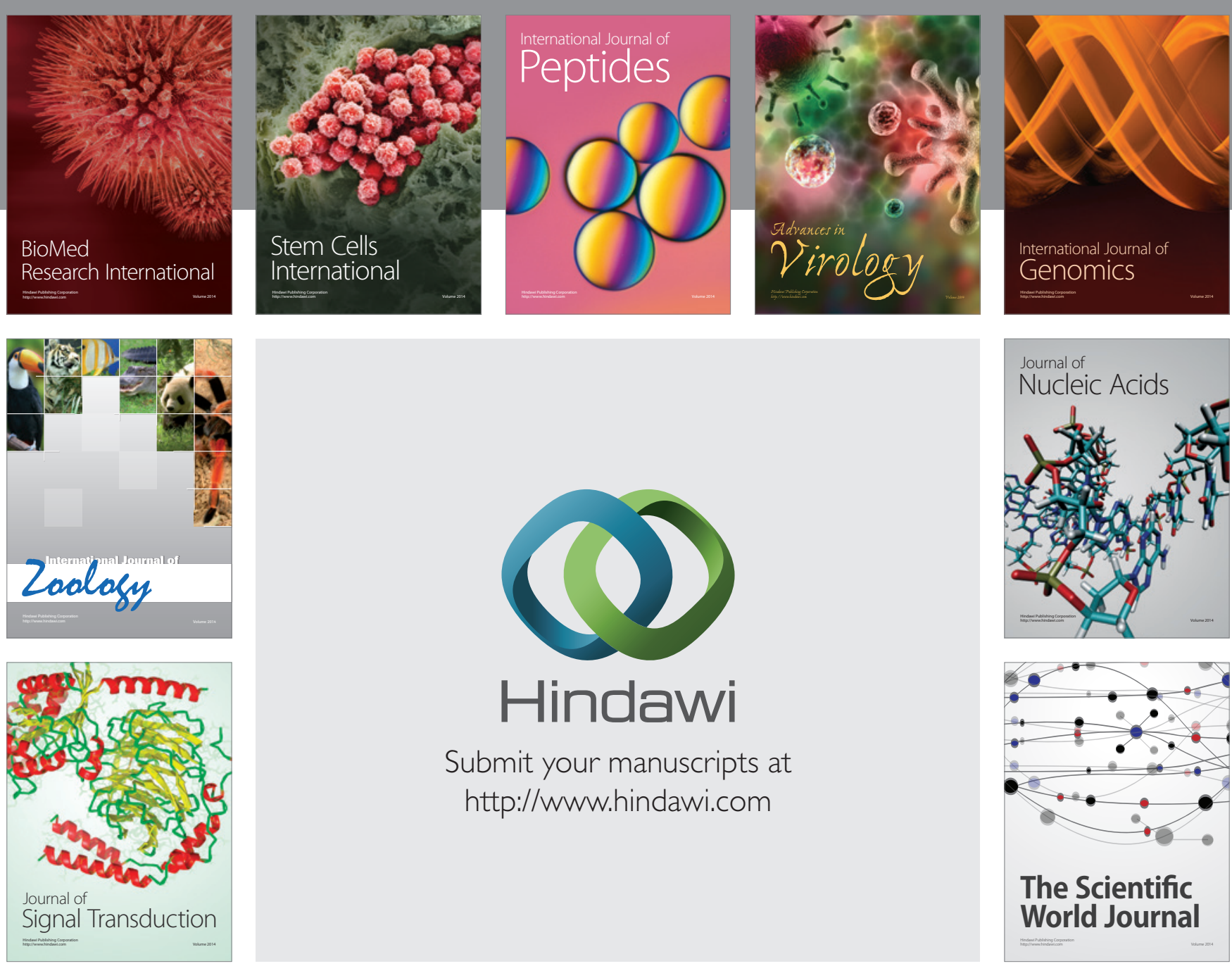

Submit your manuscripts at

http://www.hindawi.com
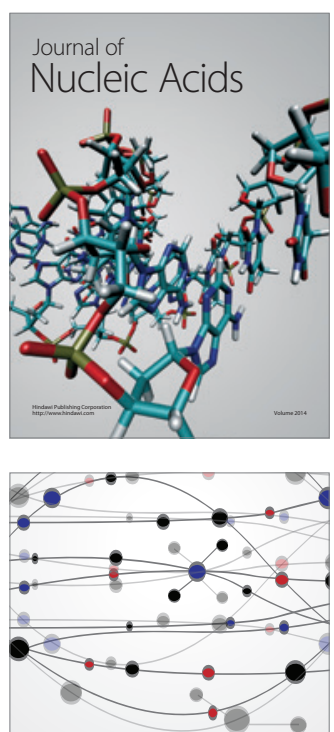

The Scientific World Journal
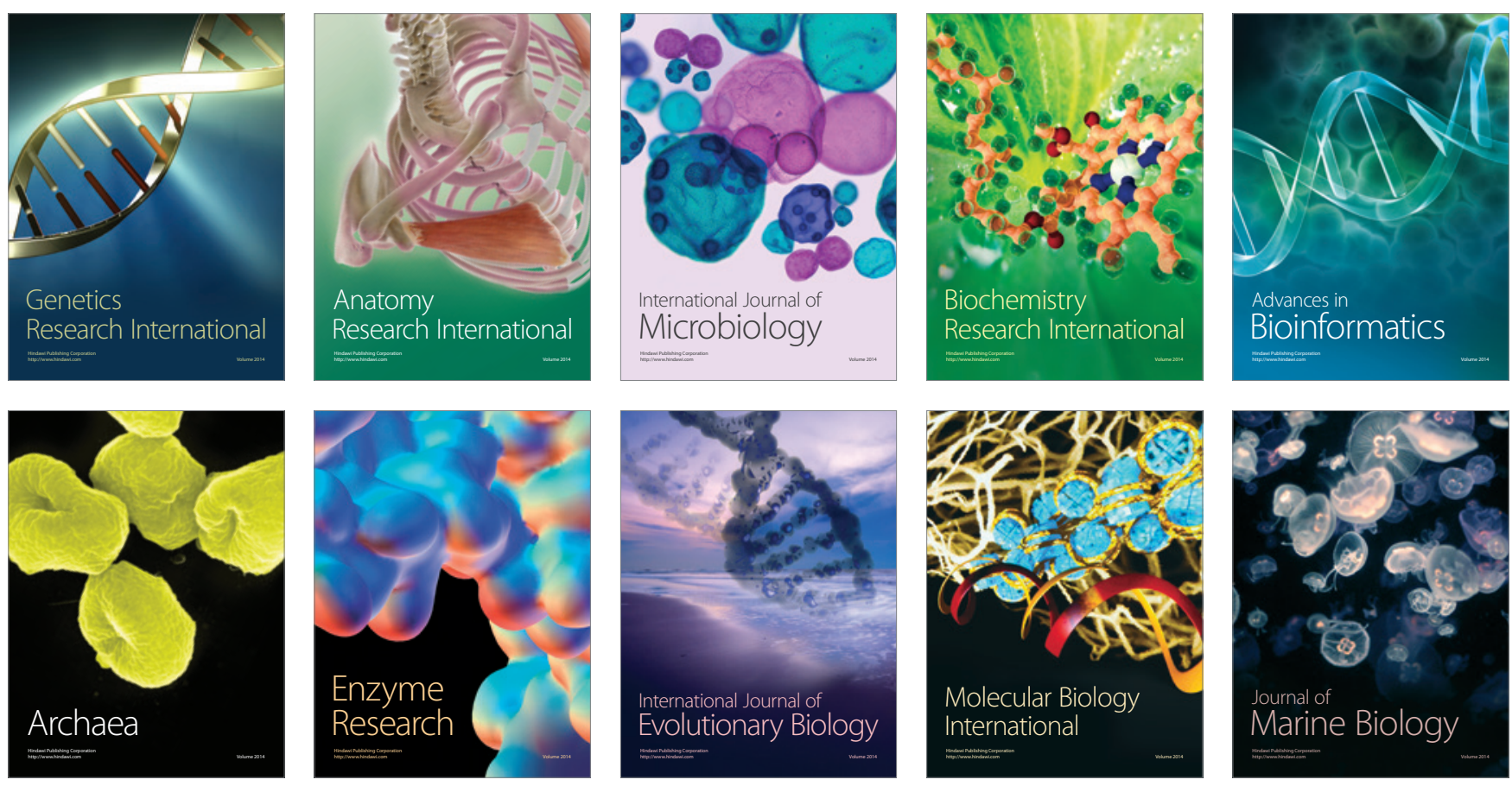